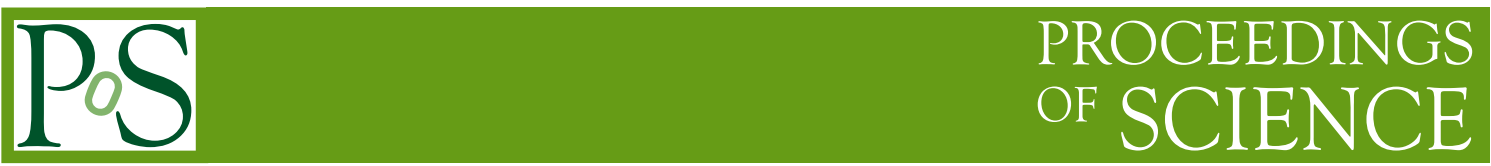

\title{
Composite Higgs scenario in mass-split models
}

\author{
Oliver Witzel, ${ }^{a, b, *}$ Anna Hasenfratz ${ }^{a}$ and Curtis T. Peterson ${ }^{a}$ \\ ${ }^{a}$ Department of Physics, University of Colorado, Boulder, CO 80309, United States \\ ${ }^{b}$ Theoretische Physik 1, Naturwissenschaftlich-Technische Fakultät, Universität Siegen, 57068 Siegen, \\ Germany \\ E-mail: oliver.witzel@uni-siegen.de, curtis.peterson@colorado.edu
}

\begin{abstract}
Mass-split composite Higgs models naturally accommodate the experimental observation of a light $125 \mathrm{GeV}$ Higgs boson and predict a large scale separation to other heavier resonances. We explore the SU(3) gauge system with four light (massless) and six heavy (massive) flavors by performing numerical simulations. Since the underlying system with degenerate and massless ten flavors appears to be infrared conformal, this system inherits conformal hyperscaling and allows to study near-conformal dynamics. Carrying out nonperturbative lattice field theory simulations, we present the low-lying particle spectrum. We demonstrate hyperscaling, predict the anomalous mass dimension of the corresponding conformal fixed point, and show that in the investigated mass regime the data are described by dilaton chiral perturbation theory. The proximity of a conformal infrared fixed point leads to a highly predictive particle spectrum which is quite distinct from QCD. Further we present initial results of our finite temperature investigations.
\end{abstract}

\section{For the Lattice Strong Dynamics collaboration}

40th International Conference on High Energy physics - ICHEP2020

July 28 - August 6, 2020

Prague, Czech Republic (virtual meeting)

\footnotetext{
${ }^{*}$ Speaker
} 


\section{Introduction}

The standard model (SM) very successfully describes the interactions of the electro-weak and strong forces. It is however an effective theory and new physics is needed to explain e.g. dark matter or UV complete the Higgs sector. Focusing at the Higgs sector, experiments have revealed a light, $125 \mathrm{GeV}$ Higgs boson [1-3] but so far no other heavier resonances. This implies that beyond the standard model (BSM) theories aiming to explain the Higgs sector are required to exhibit a large separation of scales [4-11] to match experimental observations. Such a large separation of scales occurs e.g. in near-conformal systems with a "walking" gauge coupling $[12,13]$. To explore the dynamics of near-conformal systems, we use the construction of a mass-split model built on a conformal infrared fixed point (IRFP) $[8,14,15]$. Specifically we study an SU(3) gauge system with $N_{\ell}=4$ light flavors of mass $\widehat{m}_{\ell}$ and $N_{h}=6$ heavy flavors of mass $\widehat{m}_{h}[16,17]$ using the same lattice actions as our investigation of the massless $N_{f}=10$ system $[18,19]$.

A system with ten massless flavors is conformal i.e. the gauge coupling is irrelevant and runs to the IRFP. By raising the mass of the heavy flavors, we create a mass-split system that is governed by the nearby IRFP at high energies. The heavy flavors decouple in the infrared where chiral symmetry for the light flavors breaks spontaneously and the gauge coupling starts running again. The heavy flavors play an active role in this setup. Their mass controls the separation between UV and IR scales and effectively sets the hadronic energy scale [20]. The properties of the low energy system differ significantly from a QCD-like setup. Most notably, the isosinglet scalar $\left(0^{++}\right)$appears to be light and may need to enter the effective chiral Lagrangian which requires an extension named dilaton chiral perturbation theory (dChPT) [21-26]. The particle spectrum of mass-split models is characterized by the inherited conformal hyperscaling.

In this report we first show conformal hyperscaling in our 4+6 mass-split system and extend/demonstrate dChPT to mass-split systems. In Section 3 we give a brief outlook on our studies of the underlying finite temperature phase structure. We aim to explore whether this new strongly coupled sector extending the SM could give rise to primordial gravitational waves in the early universe.

\section{Hyperscaling and Dilaton Chiral Perturbation Theory}

Properties of mass-split systems can be deduced using arguments based on Wilsonian renormalization group $(\mathrm{RG})$ methods. We assume that in the UV both mass parameters are much lighter than the cutoff $\Lambda_{\text {cut }}=1 / a$, i.e. $\widehat{m}_{l} \ll 1, \widehat{m}_{h} \ll 1$ where $a$ denotes the lattice spacing and $\widehat{m}_{\ell, h}$ are masses in lattice units. Once the energy scale $\mu$ is lowered from the cutoff, the RG flowed lattice action moves in the infinite parameter action space. Both masses increase according to their scaling dimension $y_{m}, \widehat{m}_{\ell, h} \rightarrow \widehat{m}_{\ell, h}(a \mu)^{-y_{m}}$. They remain however sufficiently small such that the system stays close to the conformal critical surface and gauge couplings run toward the IRFP.

For this scenario we can use standard hyperscaling arguments [27-29] to show that any physical quantity $a M_{H}$ of mass dimension one follows, at leading order, the scaling form [15]

$$
a M_{H}=\widehat{m}_{h}^{1 / y_{m}} \Phi_{H}\left(\widehat{m}_{\ell} / \widehat{m}_{h}\right)
$$



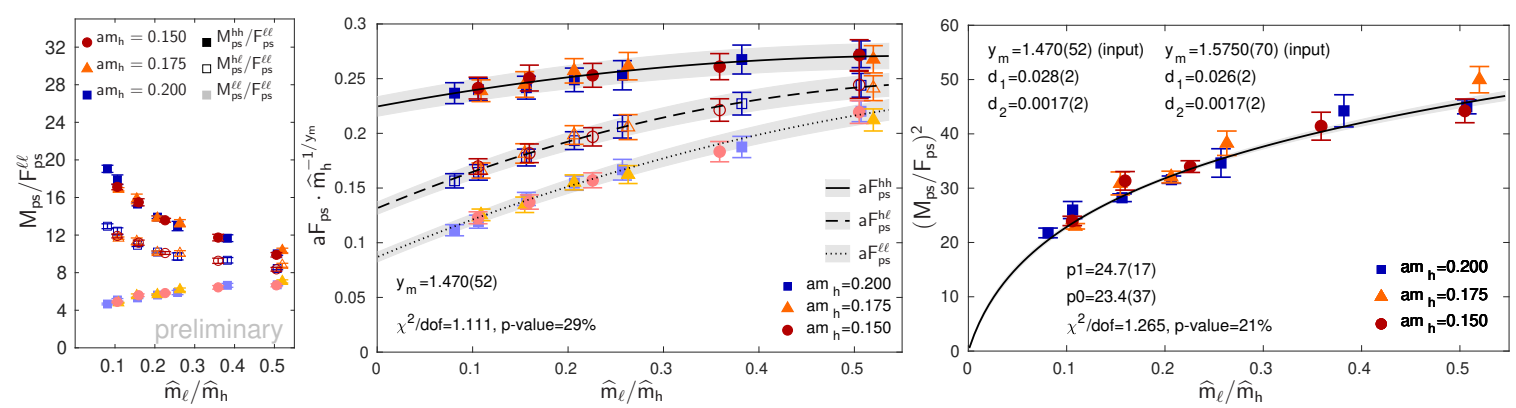

Figure 1: Left: ratio of pseudoscalar mass $\left(M_{\mathrm{ps}}\right)$ over pseudoscalar decay constant $\left(F_{\mathrm{ps}}\right)$ demonstrating hyperscaling for dimensionless ratios. Middle: combined and correlated fit using the hyperscaling relation (Eq. (1)) to extract the universal scaling dimension $y_{m}$ from measured $F_{\mathrm{ps}}$ values. Right: testing dChPT in the light sector assuming a specific form of the dilaton potential.

where $y_{m}=1+\gamma_{m}^{\star}$ is the universal scaling dimension of the mass at the IRFP and $\Phi_{H}$ some function of $\widehat{m}_{\ell} / \widehat{m}_{h}$. If we consider a ratio of two quantities, Eq. (1) implies that this ratio will only depend on $\widehat{m}_{\ell} / \widehat{m}_{h}$.

The left panel of Fig. 1 demonstrates this by showing ratios of pseudoscalar masses with two heavy, two light, or one heavy and one light flavor in the valence sector over the light-light pseudoscalar decay constant. Despite changing $m_{h}$ from 0.200 to 0.150 , data for the three different ratios (open, filled, and shaded symbols) trace out unique curves. The high quality of our data even allows us to directly exploit the hyperscaling relation in Eq. (1) as we show in the central panel. Replacing the unknown functions $\Phi_{H}$ by a polynomial of second degree, we perform a combined and correlated fit to 51 data points for the pseudoscalar decay constants. The fit exhibits an excellent $p$-value and finds the universal scaling dimension $y_{m}=1.470(42)$. Since $\gamma_{m}^{\star}$ is small, this suggests that $N_{f}=10$ is sufficiently far from the onset of the conformal window [12,30]. Our value lies in between values predicted for $N_{f}=8$ and 12 [31-39].

Next we consider the low energy infrared limit of our system. Since chiral symmetry of the light sector breaks spontaneously, we expect it can be described by a chiral effective Lagrangian smoothly connecting to the hyperscaling relation Eq. (1) which is valid at the hadronic scale $\mu=\Lambda_{H}$. First we express the lattice scale $a$ in terms of the hadronic scale $\Lambda_{H}$

$$
M_{H} / \Lambda_{H}=\left(a M_{H}\right) \cdot \widehat{m}_{h}^{-1 / y_{m}}=\Phi_{H}\left(\widehat{m}_{\ell} / \widehat{m}_{h}\right),
$$

and deduce the relation

$$
m_{f} \propto \widehat{m}_{\ell}\left(a \Lambda_{H}\right)^{-y_{m}} \cdot \Lambda_{H}=\left(\widehat{m}_{\ell} / \widehat{m}_{h}\right) \cdot \Lambda_{H} .
$$

Equation (1) and the predicted $y_{m}=1.470(42)$ scaling exponent indicate that our data do not follow the standard chiral perturbative form. We test an extension, dChPT [21-26], that assumes the presence of a light scalar state. Following [26] we make a specific assumption for the Higgs potential which leads to the relation

$$
\frac{M_{p s}^{2}}{F_{p s}^{2}}=\frac{1}{y_{m} d_{1}} W_{0}\left(\frac{y_{m} d_{1}}{d_{2}} m_{f}\right),
$$



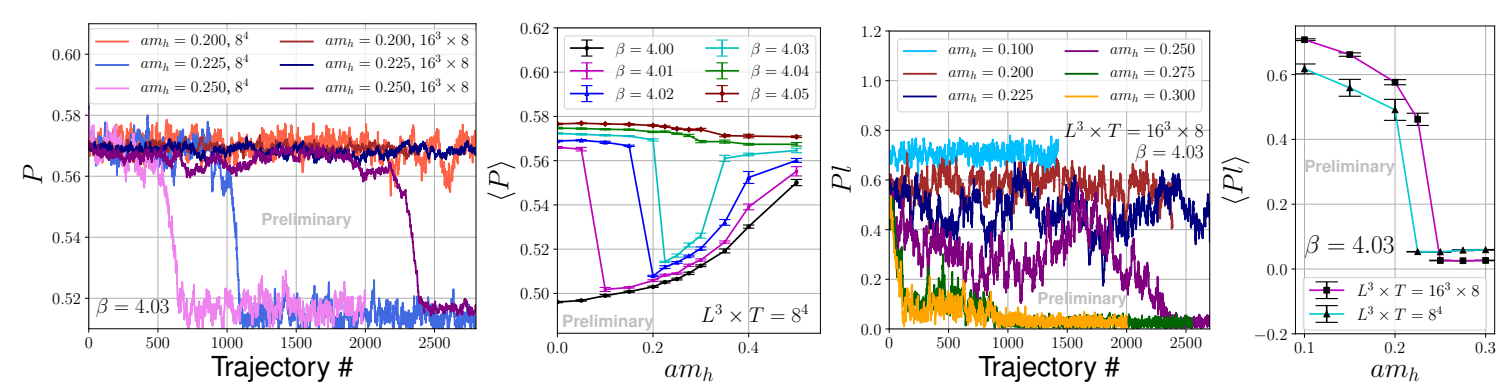

Figure 2: Left: Time history of the plaquette $P$ for $8^{4}$ and $16^{3} \times 8$ simulations at $\beta=4.03$. Middle left: Ensemble averaged plaquette against $a m_{h}$ for simulations on $8^{4}$ lattices. Middle right: Time history of Polyakov loop for $16^{3} \times 8$ lattices at $\beta=4.03$. Right: Comparison of the ensemble averaged Polyakov loop for $8^{4}$ and $16^{3} \times 8$ lattices at $\beta=4.03$ for different values of $a m_{h}$. All simulations have $\widehat{m}_{\ell} / \widehat{m}_{h}=1$.

where $W_{0}$ is the Lambert W-function and $d_{1}, d_{2}$ are mass independent constants. Using our data from the light sector, we can fit for $d_{1}$ and $d_{2}$ as shown in the right panel of Fig. 1. This further demonstrates the beauty of our mass-split model. The relevant parameter is $\widehat{m}_{\ell} / \widehat{m}_{h}$, which is a continuous variable. Since this fit works well, it can be interpreted that our simulations performed so far fall into the range of dChPT. Additional simulations at smaller $\widehat{m}_{\ell} / \widehat{m}_{h}$ may reveal the need for higher order terms or the applicability of regular chiral perturbation theory.

\section{Investigations of the Finite- and Zero-Temperature Phase Structure}

Understanding the phase structure of the $N_{f}=10$ mass-degenerate and $4+6$ mass-split systems provides insight into their continuum thermodynamic properties and their behavior as lattice models. On the one hand, the order of the finite-temperature phase transition yields information about these systems that could be relevant in the early universe. A particularly exciting possibility is the production of primordial gravitational waves from a first-order finite-temperature transition that next-generation gravitational wave detectors may unearth [40-43]. On the other hand, we also expect a zero-temperature bulk phase transition separating a weak coupling phase with a welldefined continuum limit from a strong coupling "bulk" phase [44-46]. The average plaquette $\langle P\rangle$ and average Polyakov loop $\langle P l\rangle$, despite not being true order parameters, are sensitive to both transitions [44]. We therefore utilize both observables to understand the location and order of each phase transition. In Fig. 2, we present preliminary results for the phase transitions in the $N_{f}=10$ mass-degenerate system using $16^{3} \times 8$ and $8^{4}$ lattices, where the fourth direction $\left(a L_{4}\right)$ corresponds to the inverse temperature.

We map out the bulk phase transition on zero-temperature $8^{4}$ lattices by keeping the bare gauge coupling $\beta$ fixed and varying $a m_{h}$. The left most panel in Fig. 2 shows the time history of the plaquette, the smallest $1 \times 1$ Wilson loop, at gauge coupling $\beta=4.03$. We observe that systems with $a m_{h}=0.225$ and 0.250 on $8^{4}$ volumes $\left(a m_{h}=0.250\right.$ on $\left.16^{3} \times 8\right)$ change its phase after several hundred trajectories in our Markov chain Monte Carlo simulation. The trajectory number of the transition depends on initial conditions and has no relevance. Calculating ensemble averages after thermalization effects or observed transitions, we show for the $8^{4}$ lattices in the second panel from the left, the ensemble averaged plaquette $\langle P\rangle$ as function of $a m_{h}$ for several values of the 
bare coupling $\beta$. These data reveal a first order phase transition in $a m_{h}$ from a weak to a strong coupling phase for $4.01 \lesssim \beta \lesssim 4.03$. The bulk transition weakens and turns into a crossover for $\beta \gtrsim 4.04$. Presently we are performing $16^{4}$ simulations to verify that we indeed observe a bulk phase transition.

In addition we study the Polyakov loop to explore the finite temperature phase structure. The third panel from the left shows the Monte Carlo time histories of the Polyakov loop on $16^{3} \times 8$ lattices at $\beta=4.03$. Obtaining the ensemble averages for simulations on $16^{3} \times 8$ and $8^{4}$ lattices at $\beta=4.03$, we find the finite temperature structure in the fourth panel. Near $a m_{h} \sim 0.2$ the averaged Polyakov loop for both $8^{4}$ and $16^{3} \times 8$ lattices sharply drops to zero. There is however a small shift in the value of $a m_{h}$ where this drop occurs. This requires further scrutiny. To better disentangle the finite temperature from the bulk phase transition, simulations on larger volumes with larger $L_{4}$ are required. While bulk phase transitions do not depend on the volume, finite temperature transitions will move with $L_{4}$ and hence separate.

In the future, we also plan to measure other observables, such as the chiral condensate, alongside zero- and finite-temperature simulations on larger lattices for a range of different bare gauge couplings. We are complementing these investigations studying the phase structure of masssplit systems exploring different values of the ratio of $\widehat{m}_{\ell} / \widehat{m}_{h}$.

\section{Acknowledgments}

We are very grateful to Peter Boyle, Guido Cossu, Antonin Portelli, and Azusa Yamaguchi for developing the Grid software library ${ }^{1}$ [47] which provides the basis of this work and thank Andrew Pochinsky and Sergey Syritsyn for developing Qlua ${ }^{2}$ [48] used for measurements. A.H. and O.W. acknowledge support by DOE Award No. DE-SC0010005. This material is based upon work supported by the National Science Foundation Graduate Research Fellowship Program under Grant No. DGE 1650115. We thank the Lawrence Livermore National Laboratory (LLNL) Multiprogrammatic and Institutional Computing program for Grand Challenge supercomputing allocations. We also thank Argonne Leadership Computing Facility for allocations through the INCITE program. ALCF is supported by DOE contract DE-AC02-06CH11357. Computations for this work were carried out in part on facilities of the USQCD Collaboration, which are funded by the Office of Science of the U.S. Department of Energy, the RMACC Summit supercomputer [49], which is supported by the National Science Foundation (awards ACI-1532235 and ACI-1532236), the University of Colorado Boulder, and Colorado State University and on Boston University computers at the MGHPCC, in part funded by the National Science Foundation (award OCI-1229059). We thank ANL, BNL, Fermilab, Jefferson Lab, MGHPCC, LLNL, the NSF, the University of Colorado Boulder, and the U.S. DOE for providing the facilities essential for the completion of this work.

\section{References}

[1] ATLAS collaboration, G. Aad et al., Phys.Lett. B716 (2012) 1 [1207. 7214].

[2] CMS collaboration, S. Chatrchyan et al., Phys.Lett. B716 (2012) 30 [1207 . 7235].

[3] ATLAS, CMS collaboration, G. Aad et al., Phys. Rev. Lett. 114 (2015) 191803 [1503.07589].

1https://github.com/paboyle/Grid

2https://usqcd.Ins.mit.edu/w/index.php/QLUA 
[4] R. Contino, Proceedings TASI 09 (2011) 235 [1005 . 4269].

[5] M. A. Luty and T. Okui, JHEP 09 (2006) 070 [hep-ph/0409274].

[6] D. D. Dietrich and F. Sannino, Phys. Rev. D75 (2007) 085018 [hep-ph/0611341].

[7] M. A. Luty, JHEP 04 (2009) 050 [0806.1235].

[8] R. C. Brower, A. Hasenfratz, C. Rebbi et al., Phys. Rev. D93 (2016) 075028 [1512 . 02576].

[9] C. Csaki, C. Grojean and J. Terning, Rev. Mod. Phys. 88 (2016) 045001 [1512 . 00468].

[10] N. Arkani-Hamed, R. T. D'Agnolo, M. Low et al., JHEP 11 (2016) 082 [1608. 01675].

[11] O. Witzel, PoS LATTICE2018 (2019) 006 [1901.08216].

[12] K. Yamawaki, M. Bando and K.-i. Matumoto, Phys. Rev. Lett. 56 (1986) 1335.

[13] M. Bando, T. Kugo and K. Yamawaki, Phys. Rept. 164 (1988) 217.

[14] R. C. Brower, A. Hasenfratz, C. Rebbi et al., J. Exp. Theor. Phys. 120 (2015) 423 [1410 . 4091].

[15] A. Hasenfratz, C. Rebbi and O. Witzel, Phys. Lett. B773 (2017) 86 [1609. 01401].

[16] O. Witzel, A. Hasenfratz and C. Rebbi, $\overline{1810.01850 .}$

[17] Lattice Strong Dynamics collaboration, T. Appelquist, R. C. Brower, K. K. Cushman et al., 2007.01810.

[18] A. Hasenfratz, C. Rebbi and O. Witzel, Phys. Lett. B798 (2019) 134937 [1710 . 11578].

[19] A. Hasenfratz, C. Rebbi and O. Witzel, Phys. Rev. D 101 (2020) 114508 [2004. 00754].

[20] A. Hasenfratz, C. Rebbi and O. Witzel, EPJ Web Conf. 175 (2018) 08007 [1710 . 08970].

[21] M. Golterman and Y. Shamir, Phys. Rev. D94 (2016) 054502 [1603. 04575].

[22] T. Appelquist, J. Ingoldby and M. Piai, JHEP 03 (2018) 039 [1711. 00067].

[23] T. Appelquist, J. Ingoldby and M. Piai, JHEP 07 (2017) 035 [1702 . 04410].

[24] M. Golterman and Y. Shamir, Phys. Rev. D98 (2018) 056025 [1805. 00198].

[25] T. Appelquist, J. Ingoldby and M. Piai, Phys. Rev. D 101 (2020) 075025 [1908. 00895].

[26] M. Golterman, E. T. Neil and Y. Shamir, 2003.00114.

[27] T. DeGrand and A. Hasenfratz, Phys. Rev. D80 (2009) 034506 [0906 . 1976].

[28] L. Del Debbio and R. Zwicky, Phys. Rev. D82 (2010) 014502 [1005 . 2371].

[29] L. Del Debbio and R. Zwicky, Phys. Lett. B 700 (2011) 217 [1009. 2894].

[30] S. Matsuzaki and K. Yamawaki, Phys. Rev. Lett. 113 (2014) 082002 [1311. 3784].

[31] T. Appelquist, G. Fleming, M. Lin et al., Phys.Rev. D84 (2011) 054501 [1106. 2148].

[32] T. DeGrand, Phys.Rev. D84 (2011) 116901 [1109.1237].

[33] A. Cheng, A. Hasenfratz, G. Petropoulos et al., JHEP 1307 (2013) 061 [1301.1355].

[34] A. Cheng, A. Hasenfratz, Y. Liu et al., Phys.Rev. D90 (2014) 014509 [1401.0195].

[35] M. P. Lombardo, K. Miura, T. J. N. da Silva et al., JHEP 12 (2014) 183 [1410 . 0298].

[36] T. A. Ryttov and R. Shrock, Phys. Rev. D 94 (2016) 105014 [1608. 00068].

[37] T. A. Ryttov and R. Shrock, Phys. Rev. D95 (2017) 105004 [1703. 08558].

[38] T. A. Ryttov and R. Shrock, Phys. Rev. D 97 (2018) 025004 [1710 . 06944].

[39] Z. Li and D. Poland, 2005.01721.

[40] Lattice Strong Dynamics collaboration, R. Brower, K. Cushman, G. Fleming et al., 2006. 16429.

[41] C. Caprini, M. Hindmarsh, S. Huber et al., JCAP 1604 (2016) 001 [1512 . 06239].

[42] A. J. Helmboldt, J. Kubo and S. van der Woude, Phys. Rev. D 100 (2019) 055025 [1904 . 07891].

[43] S. Schettler, T. Boeckel and J. Schaffner-Bielich, Phys. Rev. D 83 (2011) 064030 [1010 . 4857].

[44] D. Schaich, A. Cheng, A. Hasenfratz et al., PoS LATTICE2012 (2012) 028 [1207.7164].

[45] X.-Y. Jin and R. D. Mawhinney, PoS LATTICE2011 (2011) 066 [1203. 5855].

[46] A. Deuzeman, M. P. Lombardo, T. Nunes Da Silva et al., Phys. Lett. B 720 (2013) 358 [1209. 5720].

[47] P. Boyle, A. Yamaguchi, G. Cossu et al., PoS LATTICE2015 (2015) 023 [1512 . 03487].

[48] A. Pochinsky, PoS LATTICE2008 (2008) 040.

[49] J. Anderson, P. J. Burns, D. Milroy et al., Proceedings of PEARC17 (2017) . 